\title{
The effect of intensive exercise program and kinesiotaping following total knee arthroplasty on functional recovery of patients
}

\author{
Birhan Oktas ${ }^{1 *}$ (D) and Ozge Vergili²
}

\begin{abstract}
Background: Total knee arthroplasty (TKA) is a gold standard for terminal term gonarthrosis patients in order to diminish pain, correct deformities, and regain stability. Postoperative functional recovery of patients depends on the current postoperative rehabilitation program to an important extent. The purpose of our study is to compare midterm functional level of gonarthrosis patients who were included in physiotherapy rehabilitation program following TKA with people in similar ages without any surgical indication nor intervention. We have aimed also to compare functionality of people who had additional kinesiotaping (KT) treatment with people who had conservative treatment only after knee arthroplasty.

Methods: Functional level and knee functionality of people in study were evaluated at the end of postoperative first month by 6-min walk test and Lysholm knee score respectively.

Results: There was no statistical difference between groups with and without TKA in terms of height, weight, body mass index, Lysholm score, and 6-min walk test score $(p>0.05)$. In patient group with TKA, people with postoperative $K T$ treatment had greater 6 -min walk test score for postoperative first month $(p=0.005)$.

Conclusion: TKA is considered a gold standard procedure in order to increase the quality of life and improve functionality of terminal term gonarthrosis patients. It is necessary to immediately control pain and edema of knee and apply intensive rehabilitation program aimed at muscle strengthening around hip and knee in order to increase the success of operation.
\end{abstract}

\section{Background}

The knee joint is one of the major joints that take an important place in the functionality of a person in everyday life. Therefore, in the advanced stages of gonarthrosis, the quality of life of an individual considerably decreases. In this direction, total knee arthroplasty (TKA) is used as the gold standard in coping with pain, deformity, and instability, especially in terminal term gonarthrosis patients [1-3]. The reason for the fact that TKA is a procedure that can be accepted as the gold standard is that postoperative results are quite satisfactory [4]. However, the share of physiotherapy and rehabilitation programs

\footnotetext{
*Correspondence: birhanoktas@kku.edu.tr; birhanoktas@gmail.com ${ }^{1}$ Orthopaedie and Traumatology Department, Kirikkale University Faculty of Medicine, Yurtkur Sokak, No:1 Yahsihan, Kirikkale, Turkey

Full list of author information is available at the end of the article
}

applied only in the postoperative period is rather big in these results in cases in which they are initiated in the preoperative period and continued for a certain time in the postoperative period, and the patient cannot be reached in the preoperative period because it is not possible for the surgery alone to bring especially the lower extremity muscle strength to the level of a healthy population [5]. In patients in the advanced stages of gonarthrosis, the decrease in the lower extremity muscle strength already reaches a certain level in the preoperative period and progresses further in the postoperative period [6-8]. Thus, the rehabilitation of the knee joint, of which stabilization is ensured primarily by soft tissues, plays an important role in the improvement of the functional levels of patients. The place of exercise programs in rehabilitation is undoubtedly great. Exercise programs 
can be applied under the supervision and control of supervisor physiotherapists, as well as in the form of in-home programs, provided that they are taught to the patient before discharge and are revised and updated periodically [9-11]. One of the most important factors affecting the success of both surgery and preoperative rehabilitation programs is edema and pain around the knee in the early postoperative period. Edema and pain cause inadequacies in the functional capacity of the individual in the early and late postoperative period $[12,13]$. Therefore, treatment modalities for edema and pain in the early postoperative rehabilitation should not be ignored. In this respect, kinesiotaping (KT) treatment, which is practical, comfortable, and safe for the patient, is one of the complementary treatment modalities with positive clinical results $[14,15]$. KT, a treatment method developed by Kenzo Kaze [16], is used clinically for the control of pain and edema and improving motor function, especially in musculoskeletal disorders and injuries [17].

The aim of this study is to compare the midterm functional levels of gonarthrosis patients, who have been applied TKA and admitted to the physiotherapy and rehabilitation program in the postoperative period, with the individuals in the similar age group who do not have surgical indications and to perform a functional comparison of the cases additionally receiving anti-edematous KT treatment in the group included in the rehabilitation program with the cases receiving only conservative treatment.

\section{Methods}

\section{Cases}

Twenty-four patients (study group) in the age range of 52-75 years who were diagnosed with stage 4 gonarthrosis according to the Kellgren-Lawrence radiological classification and underwent unilateral cemented TKA with the medial parapatellar retinacular approach and $17 \mathrm{pa}-$ tients (control group) with no surgical indication according to this classification were included in the study. The mean age of the study and control groups was $63.3 \pm 6.9$ and $63.6 \pm 5.2$ years, the body mass indices were $28.9 \pm$ 4.0 and $29.7 \pm 4.5 \mathrm{~kg} / \mathrm{m}^{2}$, and the groups included two and one male patients, respectively. The exclusion criteria for the control group were as follows: having a value of 4 and above when the activity pain of the knee joints was questioned by a $10-\mathrm{cm}$ visual analogue scale and having various lower extremity and/or back problems (fracture history, knee tumors, disc herniation, etc.) and cognitive impairment. The exclusion criteria for the study group were as follows: having a value of 4 and above when the activity pain of the non-operated knee joint was questioned by a $10-\mathrm{cm}$ visual analogue scale, having bilateral arthroplasty, revision arthroplasty, various problems (fracture history, knee tumors, disc herniation, etc.) of operated and/or non-operated leg and/or back, developing complications secondary to the operation (deep vein thrombosis, infection, etc.), and having cognitive impairment. The study protocol was approved by Kırıkkale University, Ethics Committee for Clinical Trials, and an informed voluntary consent form was obtained from all the individuals participating in the study.

\section{Study design}

Patients who had undergone TKA were divided into two groups using the random number generator. While conservative postoperative physiotherapy program was applied to one of the groups, KT treatment was applied to the other group during a 1-week hospital stay in addition to this program. The conservative exercise program was initiated on the second postoperative day. The exercise program performed by being combined with a 20-min local cold application every $34 \mathrm{~h}$ a day was initiated with ankle pump exercises, deep breathing exercises, isometric knee and hip exercises, sitting on the edge of the bed, and short-term walking with a load on the prosthetic side at a tolerable level; the number and frequency of the exercises were increased; the isotonic knee and hip circumference strengthening exercises were performed; the program was advanced by increasing the sitting and walking distances; and finally, the patients were discharged at the end of the first week by giving ambulation training and a home exercise program. Stretching exercises were also addressed in the home exercise program, and patients were informed about the conditions and activities to be considered. In addition to this program, the lymphatic correction technique was applied to the parapatellar region using a 0 $10 \%$ stretch to reduce edema and pain from the second postoperative day in the group receiving KT treatment. As the edema was in acute phase, EDF (epidermis, dermis, fascia) technique was used by cutting the tape into as much pieces as possible in order to direct the lymphatic correction effect of tape to the superficial subcutaneous tissues predominantly.

The tapes were changed every other day, and the new ones were applied. The patients were taped for the last time before being discharged and were asked to remove the tapes 3 days after as they had been taught.

\section{Outcome measures}

The functional levels and knee functionalities of all cases included in the study were evaluated using the 6-min walk test and the Lysholm knee score, respectively. These evaluations of the TKA patients included in the study group were performed at the end of the first postoperative month. The 6-min walk test, which is an excellent tool for estimating functional ambulance [18], is frequently used clinically to evaluate the walking speed and endurance following TKA [19-21]. The 6-minute 
walk test, which is a reliable test for evaluating functionality in the patient population in question [22], is a highly reliable test that can also be used to evaluate the functional performance of patients following total hip arthroplasty [23]. The Lysholm knee scale evaluates pain, instability, locking, swelling, limping, stair climbing, crouching, and walking support need in the knee joint under eight main headings, and the Turkish version of the scale in question is a clinically feasible, valid, and reliable evaluation tool that can be used to evaluate the knee functionality in a variety of disorders of the knee joint [24] (Table 1).

\section{Statistical analysis}

Statistical analyses were performed using IBM SPSS Statistics 21 software. As descriptive statistics, number and percentage were used for qualitative data, and mean \pm standard deviation were used for numerical variables. Whether numerical variables conform with normal distribution was examined by the Shapiro-Wilk test. Whether there was a difference in terms of the qualitative variable (gender) between the two groups was examined by Fisher's exact test, and the difference in terms of numerical variables in the independent groups was analyzed by the $t$ test. The $p<0.05$ value was accepted to be statistically significant.

\section{Results}

When the groups undergoing and not undergoing TKA were considered, no difference was found between the two groups in terms of age $(t=-0.123, p=0.903)$. The gender distribution of the groups is not different ( $p=$ 1.000). There was no significant difference between the two groups in terms of body mass index (BMI), Lysholm score, and 6-min walk test score $(p>0.05)$ (Table 2). In the patient group which had undergone TKA, a difference was found in the 6-min walk test scores of individuals receiving and not receiving $\mathrm{KT}$ treatment only in the postoperative first month $(p=0.005)$ (Table 3$)$.

\section{Discussion}

Gonarthrosis is the most common joint disease, in which stopping the degenerative process is not possible. The gold standard for diminishing pain, correcting deformities, and regaining stability in terminal term patients is total knee arthroplasty (TKA) [1-3]. Following TKA, especially at the end of the second year, it has been shown that the cardiovascular compliance level of patients increases in parallel with their functionality. Thus, the TKA approach has been shown to have a positive effect on the quality and length of patients' life [25].

In patients with end-stage symptomatic knee osteoarthritis (OA), while TKA improves the physical function based on a personal report [26], a performance-based
Table 1 Lysholm knee score

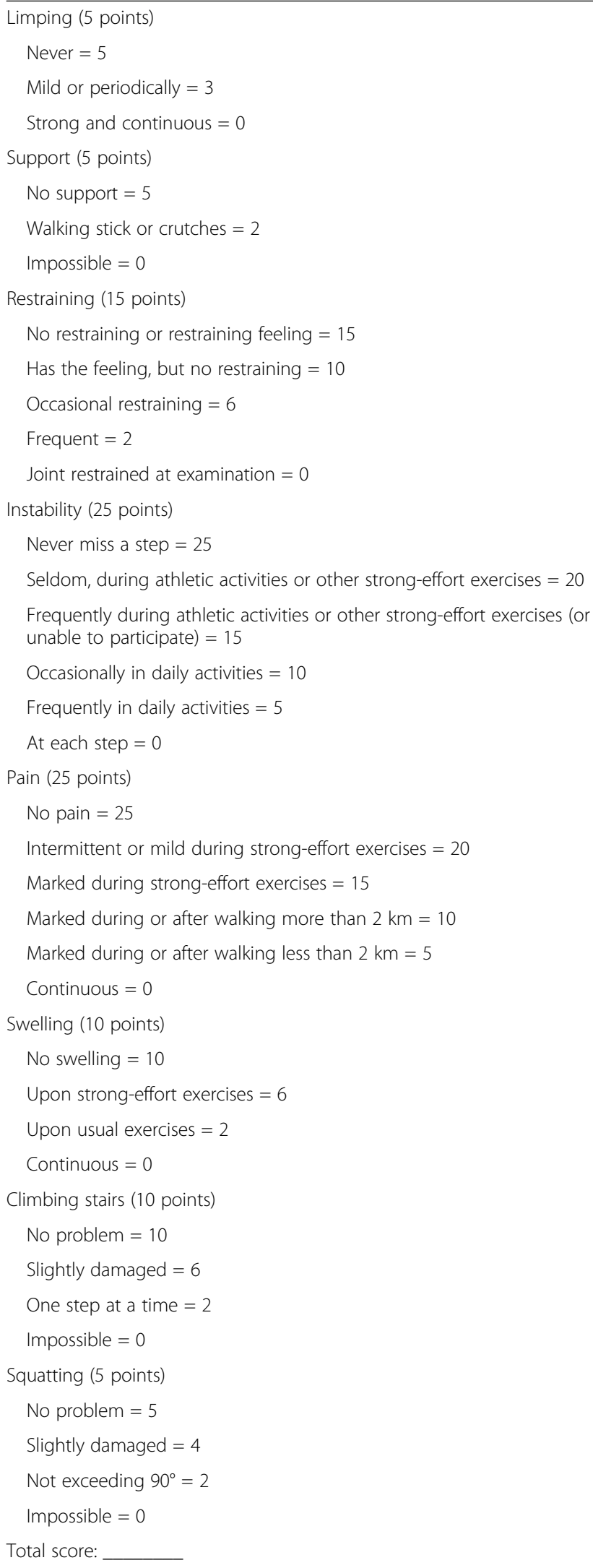


Table 2 Comparison of the patients according to applied treatment (surgery or not)

\begin{tabular}{|c|c|c|c|c|}
\hline Variables & Case $(n=18)$ & Control $(n=17)$ & Test statistics & $p$ \\
\hline Age (years) & $63.3 \pm 6.9$ & $63.6 \pm 5.2$ & -0.123 & 0.903 \\
\hline Gender M/F & $2 / 18$ & $1 / 17$ & & 1.000 \\
\hline Body mass index $\left(\mathrm{kg} / \mathrm{m}^{2}\right)$ & $28.9 \pm 4$ & $29.7 \pm 4.5$ & -0.525 & 0.603 \\
\hline Lysholm score & $87.5 \pm 8.6$ & $86.2 \pm 10.2$ & 0.398 & 0.693 \\
\hline 6-min walk test score (meter) & $364.8 \pm 63.4$ & $325.2 \pm 73.8$ & 1.704 & 0.098 \\
\hline
\end{tabular}

The comparison of the two groups undergoing and not undergoing TKA in terms of the age, gender, height, weight, body mass index (BMI), Lysholm score, and 6-min walk test score

physical function such as walking speed improves more slowly. Walking speed limitations can last even for a year or more after the surgery [27]. In a study, by Pua et al., investigating factors affecting walking speed after acute post-TKA, it was determined that the quadriceps femoris muscle strength and range of knee joint motion are important physical factors affecting walking speed, especially on the contralateral side, and fear-based cognitive factors, which result in activity limitation, also affect this physical parameter [28]. Similarly Christensen et al. underlined the importance of quadriceps muscle strength on gait impairments following TKA [29].

There are some differences in the recovery process of patients following TKA. Most of the patients experience functional deficits in the lower extremity muscles, such as weakness and slower walking speed, difficulty in climbing up and down the stairs, and standing up from a chair, compared with healthy individuals of the same age [30-32]. Therefore, it is necessary to consider other joints, especially the hip joint as an underlying cause of this functional impairment in the patient group in question. It is once again confirmed that the hip joint has to be examined considering the hip muscle strength and hip joint mechanics required for normal knee joint functioning.

The weakness in the hip abductor muscles observed in knee OA patients may persist following TKA [33]. The strength of this muscle group in post-prosthetic patients has an important effect on the faster recovery of the knee function [34]. Therefore, it will be meaningful that exercise

Table 3 Comparison of the patients according to kinesiotaping (KT) application

\begin{tabular}{lllll}
\hline Variables & KT available & KT not available & $Z$ & $p$ \\
\hline Gender (M/F) & $2 / 10$ & $0 / 6$ & -0.703 & 0.482 \\
Age & $61(54-75)$ & $67(53-74)$ & -529 \\
Body mass index & $30.6(24.3-36)$ & $24.973(23.4-34.4)$ & -2.154 & 0.031 \\
Lysholm score & $90.5(76-99)$ & $89.5(70-99)$ & -0.330 & 0.741 \\
6-min walk test score & $408(280-460)$ & $307.5(250-345)$ & -2.777 & 0.005 \\
\hline
\end{tabular}

The comparison of the patients receiving and not receiving $\mathrm{KT}$ treatment in the TKA-applied group in terms of the age, gender, height, weight, body mass index (BMI), Lysholm score, and 6-min walk test score. Only the differences between two groups are statistically significant in terms of BMI and 6-min walk test score protocols for strengthening hip muscles take place in the routine rehabilitation programs following TKA [35]. Piva et al. emphasized the importance of the hip circumference muscle strength for the functional recovery following TKA. It was shown that the strength of the hip abductor muscles following TKA affects the physical function of patients more than the demographic or anthropometric measures or the quadriceps muscle strength.

It is known that one of each three TKA patients has residual knee pain at the end of the second postoperative year and the functional capacity of the knee has inadequacies [36]. In a study, carried out by Mizner et al. to identify the time course of the restoration of inadequacies and function following TKA and which was based on the hypothesis that the correlation between the functional performance and quadriceps muscle strength is stronger than knee flexion ROM or pain, patients were evaluated in the first, second, third, and sixth months following the 6-week physiotherapy program after discharge. Patients recovered faster than expected, and long-term outcomes were found to be better than previously reported in the literature. The high correlation between the quadriceps strength and functional performance once again emphasized the importance of muscle-based approaches in the postoperative rehabilitation programs.

The recovery of the muscle strength around the knee following TKA is very difficult. The strength deficit in the quadriceps muscle can continue at the rate of $30 \%$ even at the end of the second postoperative year. A similar level of postoperative deficits in the hamstring muscle strength can also continue for years [37]. An increase is observed in the quadriceps/hamstring co-activation due to muscle deficits in the muscle groups in question. This increase disrupts the walking kinetics and kinematics and load distribution mechanism of the knee joint and results in the continuity of the degenerative process in the knee joint [38-40]. Therefore, bilateral strength and muscle activation training should be initiated in the earliest period following TKA in order to optimize the muscle firing patterns. The strength of the knee extensor muscles is very important for the patient satisfaction and functional activity restoration following TKA; therefore, quadriceps strengthening exercises 
should be focused on postoperative rehabilitation programs [41]. Especially that the hamstring muscle group is skipped in rehabilitation programs, this results in a residual functional deficit of patients. In order to optimize the muscle function and long-term rehabilitation outcomes, the improvement of the hamstring muscle strength should be emphasized as much as quadriceps in the rehabilitation program following TKA [42].

In a study conducted by Artz et al., it was shown that conventional physiotherapeutic exercise approaches performed after primary total knee replacement provided short-term improvements in the physical function, but these gains were not long term [43]. It was reported that more intensive therapeutic approaches were required for patients to achieve functionality following TKA at the level of healthy cases and that standard rehabilitation programs only allow patients to return to their functional levels in the preoperative period [44].

Merk et al. conducted a study on rehabilitation programs following TKA; it was emphasized that sports-specific therapy was required following the conventional rehabilitation program in order to prolong the functional life of the prosthesis and to prevent falls and inappropriate mechanical stress on the joint and that weakness in the quadriceps muscle group would persist with the application of only conventional rehabilitation [45]. Neuromuscular electrical stimulation and higher intensity strength training programs should be considered in addition to the traditional rehabilitation approaches for accelerating the functional recovery of patients following TKA. In cases when optimal results are still not achieved, aquatic exercises and eccentric strengthening training should be focused on [46]. Bade and Stevens-Lapsley showed in their clinical study that a high-intensity rehabilitation program following TKA improves both short-term and long-term functionality and quadriceps muscle strength compared to a low-intensity one, and the high-intensity program does not cause any complications [47].

Postoperative edema and pain are important factors affecting the success of rehabilitation. It has been concluded that decreased knee extension strength, which leads to a decline in the functional performance following TKA, may be due to postoperative edema around the knee, and the edema control in the early postoperative period in rehabilitation protocols will positively affect the success of rehabilitation and the early functionality level [48].

In the acute postoperative period following TKA, both cold and kinesiotaping applications reduce pain and swelling, thus increasing the functional performance. Yüksel et al. showed that although these modalities have no advantage over each other, they have no acute effects on the muscle strength [49].
Another study by Donec et al. showed that the KT applied within the scope of an early rehabilitation program following TKA reduced postoperative pain and edema and increased the range of motion of the knee extension [50].

Deniz et al. found KT application to be an effective rehabilitative approach for relieving pain and improving function in the acute stage of TKA as they found manual lymphatic drainage to be [51].

In the musculoskeletal problems of lower extremities, $\mathrm{KT}$ was found to have positive effects on pain, edema, and specific venous symptoms, but no evidence was found for its effects on the muscle strength, health-related quality of life, and joint function [52].

\section{Conclusion}

In the present study conducted in the light of this information, it was found out that the rehabilitation program, which we initiated in the early postoperative period following TKA, improved the functional recovery of patients in a positive way and that at the end of the first postoperative month, prosthetic patients were found to have similar knee functionalities with non-prosthetic patients. We think that in the obtainment of this outcome, the fact that the exercise program that we applied ensured the necessary concentric and eccentric loading on the hip circumference muscles, and also on the quadriceps and hamstring muscles, has a great share. Our findings were supported by Pozzi et al. who underlined the importance of strengthening exercises in rehabilitation program following TKA [53]. Again, with the cold application initiated immediately after the surgery, postoperative edema and pain were taken under control early, and reflex muscle inhibition due to surgery was prevented, and thus, the functional recovery of patients was accelerated by increasing compliance and participation in exercise programs. Pain and edema control was achieved in a shorter time in TKA patients who received KT treatment in addition to cold application, and these patients were found to have higher walking distances of the 6-min walk test in the first postoperative month than those who did not receive KT treatment. Thus, the necessity of the inclusion of KT in the clinic following TKA from the early postoperative period has been revealed once again.

\section{Limitations of the study}

The number of individuals per groups is not the ones desired by the researches. It can be attributed to wide range of exclusion criteria and patients' voluntary basis nature of our study. Secondly, radiological evaluation of knees of individuals in the control group could have given a chance to understand the level of arthritic changes in their joint. Finally, knee scales in addition to 
Lysholm knee score could have been used to evaluate the functional status of individuals.

\section{Abbreviations}

KT: Kinesiotaping; OA: Osteoarthritis; TKA: Total knee arthroplasty

\section{Acknowledgements}

Thanks for permissions for conducting this study were given to our chairman Dr. Meric Cirpar and Dr. Arzu Daskapan.

\section{Availability of data and materials}

The datasets during and/or analyzed during the current study are available from the corresponding author on reasonable request.

\section{Authors' contributions}

The study is conducted by OV and BO. All arthroplasty surgeries are made by $\mathrm{BO}$, whereas all measurements and evaluations are made by $\mathrm{OV}$. The manuscript is written by two authors in cooperation. Both authors read and approved the final manuscript.

\section{Ethics approval and consent to participate}

We have ethics approval from Kirikkale University Clinical and Pharmacological Ethical Committee in our institute with the number of 26/09.

\section{Consent for publication}

All participants including patients and control group have permitted to share and publish their information with their own willingness via a declaration form signed by themselves.

\section{Competing interests}

The authors declare that they have no competing interests.

\section{Publisher's Note}

Springer Nature remains neutral with regard to jurisdictional claims in published maps and institutional affiliations.

\section{Author details}

'Orthopaedie and Traumatology Department, Kirikkale University Faculty of Medicine, Yurtkur Sokak, No:1 Yahsihan, Kirikkale, Turkey. ${ }^{2}$ Physiotherapy and Rehabilitation Department, Kirikkale University Faculty of Health Sciences, Kirikkale, Turkey.

Received: 22 May 2018 Accepted: 22 August 2018

Published online: 12 September 2018

\section{References}

1. Callahan CM, Drake BG, Heck DA, et al. Patient outcomes following tricompartmental total knee replacement. A meta-analysis. JAMA. 1994;271: 1349-57.

2. Jones CA, Beaupre LA, Johnston DW, et al. Total joint arthroplasties: current concepts of patient outcomes after surgery. Rheum Dis Clin N Am. 2007:33: 71-86.

3. Carr AJ, Robertsson O, Graves S, Price AJ, Arden NK, Judge A, Beard DJ. Knee replacement. Lancet. 2012;379:1331-40.

4. Van Herck P, Vanhaecht K, Deneckere S, et al. Key interventions and outcomes in joint arthroplasty clinical pathways: a systematic review. J Eval Clin Pract. 2010;16:39-49.

5. Hopkins JT, Ingersoll CD. Arthrogenic muscle inhibition: a limiting factor in joint rehabilitation. J Sport Rehabil. 2000;9:135-59.

6. Stevens-Lapsley JE, Balter JE, Wolfe P, et al. Early neuromuscular electrical stimulation to improve quadriceps muscle strength after total knee arthroplasty: a randomized controlled trial. Phys Ther. 2012;92:210-26.

7. Morita S, Kusaka T, Tanaka S, et al. The relationship between muscle weakness and activation of the cerebral cortex early after unicompartmental knee arthroplasty. J Phys Ther Sci. 2013;25:301-7.

8. Mizner RL, Petterson SC, Stevens JE, et al. Early quadriceps strength loss after total knee arthroplasty. The contributions of muscle atrophy and failure of voluntary muscle activation. J Bone Joint Surg Am. 2005;87:1047-53.
9. Schenck RC Jr, Blaschak MJ, Lance ED, et al. A prospective outcome study of rehabilitation programs and anterior cruciate ligament reconstruction. Arthroscopy. 1997;13:285-90.

10. Wheatley WB, Krome J, Martin DF. Rehabilitation programmes following arthroscopic meniscectomy in athletes. Sports Med. 1996;21:447-56.

11. Rajan RA, Pack Y, Jackson $H$, et al. No need for outpatient physiotherapy following total knee arthroplasty: a randomized trial of 120 patients. Acta Orthop Scand. 2004;75:71-3.

12. Korean Knee Society. Guidelines for the management of postoperative pain after total knee arthroplasty. Knee Surg Relat Res. 2012;24:201-7.

13. Holm B, Kristensen MT, Bencke J, Husted H, Kehlet H, Bandholm T. Loss of knee extension strength is related to knee swelling after total knee arthroplasty. Arch Phys Med Rehabil. 2010;91:1170-776.

14. Yüksel E, Ünver B, Karatosun V. Comparison between kinesiotaping and cold therapy on muscle strength functional performance outcomes after total knee arthroplasty: preliminary results of a randomised controlled trial. Ann Rheum Dis. 2016;75:1307.2-1307. https://doi.org/10.1136/annrheumdis2016eular.6073.

15. Donec V, Crisciunas A. The effectiveness of Kinesiotaping after total knee replacement in early postoperative rehabilitation period. A randomized controlled trial. Eur J Phys Rehabil Med. 2014;50:363-71.

16. Kase K, Wallis J, Kase T. Clinical therapeutic applications of the Kinesio taping method. 2nd ed. Tokyo: Ken Ikai Co Ltd; 2003.

17. Thelen MD, Dauber JA, Stoneman PD. The clinical efficacy of kinesio tape for shoulder pain: a randomized, double-blinded. Clinical Trial J Orthop Sports Phys Ther. 2008;38:389-95

18. Ko V, Naylor JM, Harris IA, Crosbie J, Yeo AE. The six-minute walk test is an excellent predictor of functional ambulation after total knee arthroplasty. BMC Musculoskelet Disord. 2013;14:145. http://www.biomedcentral.com/ $1471-2474 / 14 / 145$

19. Kennedy DM, Stratford PW, Riddle DL. Assessing recovery and establishing prognosis following total knee arthroplasty. Phys Ther. 2008:88:22-32.

20. Bade MJ, Kohrt WM, Stevens-Lapsley JE. Outcomes before and after total knee arthroplasty compared to healthy adults. J Orthop Sports PhysTher. 2010;40:559-67.

21. Petterson SC, Mizner RL, Stevens JE. Improved function from progressive strengthening interventions after total knee arthroplasty: a randomized clinical trial with an imbedded prospective cohort. Arthritis Rheum. 2009;61:174-83.

22. Jakobsen TL, Kehlet H, Bandholm T. Reliability of the 6-min walk test after total knee arthroplasty. Knee Surg Sports Traumatol Arthrosc. 2013;21:2625-8.

23. Ünver B, Kahraman T, Kalkan S, Yüksel E, Karatosun V. Reliability of the sixminute walk test after total hip arthroplasty. Hip Int. 2013;23:541-5.

24. Çelik D, Coşkunsu D, Kılıçoğlu Ö. Translation and cultural adaptation of the Turkish Lysholm knee scale: ease of use, validity, and reliability. Clin Orthop Relat Res. 2013;471:2602-10.

25. Ries MD, Philbin EF, Groff GD. Improvement in cardiovascular fitness after total knee arthroplasty. J Bone Joint SurgAm. 1996;78A:1696-701.

26. Hamel MB, Toth M, Legedza A, Rosen MP. Joint replacement surgery in elderly patients with severe osteoarthritis of the hip or knee: decision making, postoperative recovery and clinical outcomes. Arch Intern Med. 2008;168:1430-40.

27. Walsh M, Woodhouse LJ, Thomas SG, Finch E. Physical impairments and functional limitations: a comparison of individuals1year after total knee arthroplasty with control subjects. Phys Ther. 1998;78:248-58.

28. Pua YH, Seah FTJ, Clark RA, Poon CLL, Tan JWM, Chong HC. Factors associated with gait speed recovery after total knee arthroplasty: a longitudinal study. Semin Arthritis Rheum. 2017;46:544-51.

29. Christensen C, Mizner RL, Foreman KB, Marcus R, Pelt CE, Lastayo PC. Quadriceps weakness preferentially predicts detrimental gait compensations among common impairments after total knee arthroplasty. J Orthop Res. 2018; https://doi.org/10.1002/jor.23894.

30. Schache MB, McClelland JA, Webster KE. Lower limb strength following total knee arthroplasty: a systematic review. Knee. 2014;21:12-20.

31. Mizner RL, Petterson SC, Snyder-Mackler L. Quadriceps strength and the time course of functional recovery after total knee arthroplasty. J Orthop Sports Phys Ther. 2005:35:424-36.

32. Rossi MD, Hasson S, Kohia M, Pineda E, Bryan W. Mobility and perceived function after total knee arthroplasty. J Arthroplast. 2006;21:6-12.

33. Alnahdi AH, Zeni JA, Snyder-Mackler L. Hip abductor strength reliability and association with physical function after unilateral total knee arthroplasty: a cross-sectional study. Phys Ther. 2014;94:1154-62. 
34. Piva SR, Teixeira PE, Almeida GJ, Gil AB, DiGioia IIIAM, Levison TJ. Contribution of hip abductor strength to physical function in patients with total knee arthroplasty. Phys Ther. 2011;91:225-33.

35. Schache MB, McClelland JA, Webster KE. Does the addition of hip strengthening exercises improve outcomes following total knee arthroplasty? A study protocol for a randomized trial. BMC Musculoskelet Disord. 2016;17:259.

36. Nashi N, Hong CC, Krishna L. Residual knee pain and functional outcome following total knee arthroplasty in osteoarthritic patients. Knee Surg Sports Traumatol Arthrosc. 2015;23:1841-7.

37. Silva M, Shephard EF, Jackson WO, Pratt JA, Mcclung CD, Schmalaried TP. Knee strength after total knee arthroplasty. J Arthroplast. 2003;18:605-11.

38. Rudolph KS, Schmitt LC, Lewek MD. Age-related changes in strength, joint laxity, and walking patterns: are they related to knee osteoarthritis? Phys Ther. 2007;87:1422-32.

39. Schmitt LC, Rudolph KS. Muscle stabilization strategies in people with medial knee osteoarthritis: the effect of instability. J Orthop Res. 2008;26: $1180-5$.

40. Thomas AC, Judd DL, Davidson BS, Eckhoff DG, Stevens-Lapsey JE. Quadriceps/hamstrings co-activation increases early after total knee arthroplasty. Knee. 2014;21:1115-9.

41. Furu M, Ito H, Nishikawa TS, Nankaku M, Kuriyama S, Ishikawa M, Nakamura S, Azukizawa M, Hamamoto Y, Matsuda S. Quadriceps strength affects patient satisfaction after total knee arthroplasty. J Orthop Sci. 2016;21:38-43.

42. Stevens-Lapsley JE, Balter JE, Kohrt WM, Eckhoff DG. Quadriceps and hamstrings muscle dysfunction after total knee arthroplasty. Clin Orthop Relat Res. 2010;468:2460-8.

43. Artz N, Elvers KT, Lowe CM, Sackley C, Jebson P, Beswick AD. Effectiveness of physiotherapy exercise following total knee replacement: systematic review and meta-analysis. BMC Musculoskelet Disord. 2015;16:15. https://doi. org/10.1186/s12891-015-0469-6.

44. Bade MJ, Kohrt WM, Stevens-Lapsey JE. Outcomes before and after total knee arthroplasty compared to healthy adults. J Orthop Sports Phys Ther. 2010;40:559-67.

45. Merk J, Winkler C, Best R. Joint function and strength in patients before and after knee replacement surgery. DEUTSCHE ZEITSCHRIFT FUR SPORTMEDIZIN. 2008:59:136-40.

46. Bade MJ, Stevens-Lapsley JE. Restoration of physical function in patients following total knee arthroplasty: an update on rehabilitation practices. Curr Opin Rheumatol. 2012;24:208-14.

47. Bade MJ, Stevens-Lapsley JE. Early high-intensity rehabilitation following total knee arthroplasty improves outcomes. J Orthop Sports Phys Ther 2011:41:932-41.

48. Curry AL, Goehring MT, Bell J, Jette DU. Effect of physical therapy interventions in the acute care setting on function, activity and participation after total knee arthroplasty: a systematic review. JACPT. 2018;9(3):93-106.

49. Yuksel E, Unver B, Karatosun V. Comparison between Kinesiotaping and cold therapy on muscle strength functional performance outcomes after total knee arthroplasty: preliminary results of a randomized controlled trial. Ann Rheum Dis. 2016;75 https://doi.org/10.1136/annrheumdis-2016-eular. 6073

50. Donec $V$, Kriščiunas A. The effectiveness of Kinesio Taping after total knee replacement in early postoperative rehabilitation period. A randomized controlled trial. Eur J Phys Rehabil Med. 2014;50:363-71.

51. Deniz HG, Kınıkı Gl, Oral S, Sevinç C, Çağlar O, Yüksel I. Comparison of kinesio tape application and manual lymphatic drainage on lower extremity oedema and functions after total knee arthroplasty. Ann Rheum Dis. 2018; https://doi.org/10.1136/annrheumdis-2018-eular.5452.

52. Resende RFR, Roriz P. The effects of Kinesio Taping ${ }^{\circledR}$ in lower limb musculoskeletal disorders: a systematic review. Int J Ther Rehab Res. 2017;6: $1-13$.

53. Pozzi F, White DK, Synder-Mackler L, Zeni JA. Restoring physical function after the replacement: a cross sectional comparison of progressive strengthening vs standard physical therapy. Physiother Theory Pract. 2018; https://doi.org/10.1080/09593985.2018.1479475.

\section{Ready to submit your research? Choose BMC and benefit from:}

- fast, convenient online submission

- thorough peer review by experienced researchers in your field

- rapid publication on acceptance

- support for research data, including large and complex data types

- gold Open Access which fosters wider collaboration and increased citations

- maximum visibility for your research: over $100 \mathrm{M}$ website views per year

At BMC, research is always in progress.

Learn more biomedcentral.com/submissions 